\title{
When is the discretization of a spatially distributed system good enough for control? ${ }^{\star}$
}

\author{
Bryn L1. Jones a Eric C. Kerrigan ${ }^{\text {b,c }}$ \\ ${ }^{\mathrm{a}}$ The Scottish Association for Marine Science, Scottish Marine Institute, Oban, Argyll, PA37 1QA, U.K. \\ ${ }^{\mathrm{b}}$ Department of Electrical and Electronic Engineering, Imperial College London, SW7 2AZ, U.K. \\ ${ }^{\mathrm{c}}$ Department of Aeronautics, Imperial College London, SW7 2AZ, U.K.
}

\begin{abstract}
This paper describes a new and straightforward method for controlling spatially distributed plants based on low-order models obtained from spatial discretization techniques. A suitable level of discretization is determined by computing the sequence of $v$-gaps between weighted models of successively finer spatial resolution, and bounding this by another sequence with an analytic series. It is proved that such a series forms an upper bound on the $v$-gap between a weighted model in the initial sequence and the spatially distributed weighted plant. This enables the synthesis, on low-order models, of robust controllers that are guaranteed to stabilize the actual plant, a feature not shared by most model reduction methods where the gap between the high-order model and plant is often not known, and where the gap between high-order and reduced models may be too expensive to compute. Since the calculation of the current bound is based on weighted models of small state-dimension, the new method avoids the numerical problems inherent in large-scale model reduction based approaches. The ideas presented in this paper are demonstrated on a disturbance rejection problem for a 1D heat equation.
\end{abstract}

Key words: Distributed parameter systems; partial differential equations; infinite-dimensional systems; discretization; measures of model fit; achievable controller performance.

\section{Notation}

In this paper $\mathbb{N}, \mathbb{R}$ and $\mathbb{C}$ denote the natural, real and complex fields, respectively, and $s \in \mathbb{C}$ is a complex variable. $\mathscr{L}_{2}[0, \infty)$ is the time-domain Lebesgue space of all signals of bounded energy supported on $[0, \infty)$, with norm $\|\cdot\|_{2} . \mathscr{H}_{\infty}$ is the Hardy space of all transfer functions of stable, linear, time-invariant, continuous-time systems with norm $\|\cdot\|_{\infty}$, and $\mathscr{R} \mathscr{H}_{\infty}$ denotes the subspace of rational functions in $\mathscr{H}_{\infty}$.

Throughout this paper, signals and systems in the time and frequency domains will be considered. Signals/systems that are spatially distributed will be shown in bold by lower/upper case letters, respectively, whilst those that are not spatially distributed (or lumped) will be denoted in regular font. Where it exists, the transfer function of $\mathbf{P}$

\footnotetext{
* This work was supported by the EPSRC. Corresponding author E. C. Kerrigan. Tel. +44-(0)20-759-45139/6343. Fax +44-(0)207594-6282.

Email addresses: bryn.jonesesams.ac.uk (Bryn Ll. Jones), e.kerrigandimperial.ac.uk (Eric C. Kerrigan).
}

is denoted by $\boldsymbol{P}$. If $P(s) \in \mathscr{R}^{p \times m}$, where $\mathscr{R}^{p \times m}$ is the set of real rational transfer function matrices with $p$ outputs and $m$ inputs, then $P(s)^{*}=P(-s)^{T}$ is its conjugate transpose. The ordered pairs $(N, M)$ and $(\tilde{N}, \tilde{M})$ denote normalised right and left coprime factorisations of $P$, respectively, where $N, \tilde{N}, M, \tilde{M} \in \mathscr{R} \mathscr{H}_{\infty}$. Normalised right and left graph symbols for $P$ are defined as $G:=\left[\begin{array}{l}N \\ M\end{array}\right]$ and $\tilde{G}:=\left[\begin{array}{c}-\tilde{M} \\ \tilde{N}\end{array}\right]$, respectively.

The order of convergence of sequences will also be discussed. The sequence $\left\{a_{n}\right\}$ converges to $\zeta \in \mathbb{R}$ with or$\operatorname{der} \alpha>0$ if there exists a constant $k \in \mathbb{R}_{+}$and $n_{0} \in \mathbb{N}$ such that $\left|a_{n}-\zeta\right| \leq k n^{-\alpha}$ for all $n \geq n_{0}$.

\section{Introduction}

Many systems of engineering importance are governed by partial differential equations (PDEs) in one or more spatial dimensions, and are therefore infinite-dimensional. Control of infinite-dimensional systems (of which spatially distributed/distributed parameter systems are a special case) can appear a daunting task, especially given the sophisticated mathematical apparatus devoted to the subject, e.g. Curtain \& Zwart (1995). However, countless case studies exist 
demonstrating successful control of these systems based on approximate models of finite (and typically small) state dimension.

As a simple example consider an inverted pendulum free to rotate around an axle. The pendulum motion is governed by a PDE since the mass elements are continuously distributed along the pendulum, with some flexibility between each element. Intuitively, if the pendulum is stiff then an approximation can be obtained by modelling the pendulum as a single rigid body. Describing this approximate model as 'good' or 'bad' depends on one's objectives. If the purpose of the model is to simulate tip deflection very accurately and the pendulum is slightly flexible, then it is probably not a good model. However, if the model is to be used for designing a controller to stabilize the pendulum around its vertical equilibrium, then it is likely to be a good model (as countless lab demonstrations will verify).

According to Reinschke (1999), in the control literature on linear, time-invariant, spatially distributed systems, there are approximately three different approaches for controller design:

Finite-dimensional model approach. This is the most common approach, whereby a (finite-dimensional) simulation model of the plant is obtained after performing some kind of spatial discretization (e.g. finite differencing) of the spatially distributed plant. Standard tools from control theory, such as balanced truncation (Zhou, Doyle \& Glover 1996), LQR synthesis (Skogestad \& Postlethwaite 2005), etc., can then be applied to design finite-dimensional controllers. This approach (also known as indirect controller design or early lumping) suffers from a number of drawbacks. Firstly, there are no prior guarantees that the controller designed on the simulation model will work on the actual plant, owing to a phenomenon known as spillover (Balas 1978) whereby the controller derived from a (finite-dimensional) model may fail to stabilize the actual plant. Systems with infinitely many poles either on, or asymptoting to the imaginary axis, are known to display spillover effects (Curtain $\&$ Morris 2009). Secondly, the size of the resulting finitedimensional system may be too large to directly synthesize a controller, thus necessitating some kind of model reduction. Model reduction of large-scale systems is an active research field, e.g. (Antoulas 2005, Jaimoukha \& Kasenally 1994). However, assuming a numerically reliable and efficient method is available for the system in hand, one might be able to reduce the state dimension of the system to a size amenable for controller synthesis. Proper Orthogonal Decomposition (POD) based methods appear promising in this respect, e.g. Willcox \& Peraire (2002), Rowley (2005). Again, however, there are likely to be no guarantees that the (typically open-loop) model reduction technique will not neglect important closed-loop dynamics. As an example, models obtained from Hankel norm model reduction may not be suitable for robust control design since perturbations vanishingly small in the Hankel norm can lead to instability (Jonckheere, Safanov \& Silverman 1981).
Distributed state-space and semigroup approach. Here, the PDEs describing the plant are transformed to a distributed state-space representation $\Sigma(\mathbf{A}, \mathbf{B}, \mathbf{C}, D)$ where the operator $\mathbf{A}$ generates a strongly continuous semigroup. In this framework standard design methods are generalised to spatially distributed systems (Curtain \& Zwart 1995). Although rigorous, this approach has its drawbacks. Firstly, one must prove that $\mathbf{A}$ generates a $C_{0}$-semigroup for a given statespace, which is a non-trivial task. Secondly, problems of boundary control and observation require considerable care, and thirdly, for complicated spatially distributed systems (e.g. fluid-mechanical (Aamo \& Krstic 2003)), obtaining a finite-dimensional plant model via spatial discretization may be the only practical option for controller design.

Infinite-dimensional transfer matrix approach. This is a frequency-domain approach whereby the spatial distributions of the sensors and actuators are assumed to be known and are considered part of the plant. In the transfer function domain, the plant maps the input signal $u(s) \in \mathbb{C}^{m}$ to the output signal $y(s) \in \mathbb{C}^{p}$ via the relationship $y(s)=\boldsymbol{P}(s) u(s)$, where $\boldsymbol{P}(s)$ is an integral operator whose kernel is the Laplace transform of the plant's Green's Function. Given $\boldsymbol{P}$, a finite-dimensional approximation $P \in \mathscr{R}^{p \times m}$ is calculated, together with the $\mathscr{H}_{2}$-gap (Georgiou \& Smith 1992) between them, $\delta_{g}(\boldsymbol{P}, P)=\vartheta$, where $0 \leq \vartheta \leq 1$. A finitedimensional controller $C(s)$ is then computed that achieves satisfactory levels of stability and performance for any plant model $P_{i}$ satisfying $\delta_{g}\left(P_{i}, P\right) \leq \vartheta$ (Reinschke 1999, Reinschke \& Smith 2003). Currently, the main drawback to this approach is that it requires the plant's Green's Function in order to obtain $\delta_{g}(\boldsymbol{P}, P)$ and deriving Green's Functions is, in general, a non-trivial task.

The techniques employed in this paper are adopted from this last approach but avoid the need for deriving Green's Functions. A less conservative notion of distance between LTI systems than the $\mathscr{H}_{2}$-gap metric is also used. For simplicity, the spatial locations of sensors and actuators are considered to be fixed; if this is not the case, then the methodology of Reinschke (1999) can be applied to determine these locations as part of the controller. Section 3 of this paper describes the mathematical tools and framework required in later sections. Section 4 describes the design procedure for controlling a spatially distributed plant, together with the relevant theoretical justification, and Section 5 applies this design process to a disturbance rejection problem for the 1-D heat equation.

\section{Preliminaries on Linear Systems}

In this paper, systems are considered from an input-output point of view, whereby the system can be thought of as an operator that maps an input space to an output space. The systems considered in this paper are linear, time-invariant (LTI) and infinite dimensional in one or more spatial dimensions (spatially distributed). In this section the relevant concepts and results are summarised from Vinnicombe (2001) 


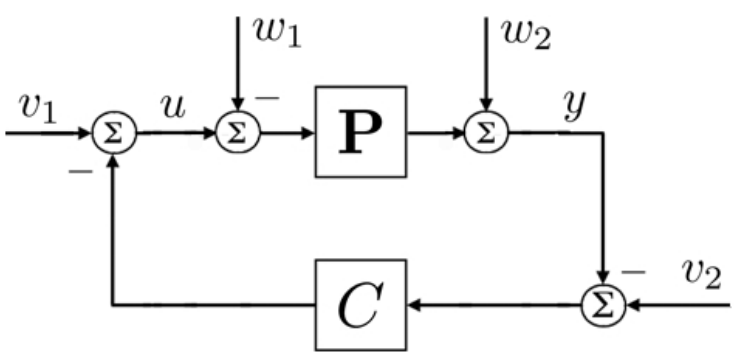

Fig. 1. Standard feedback configuration.

and Georgiou \& Smith (1992).

In Figure 1, let the plant $\mathbf{P}$ be the linear mapping $\mathbf{P}: \mathscr{D}(\mathbf{P}) \subset$ $\mathscr{U} \rightarrow \mathscr{Y}$ where $\mathscr{U}:=\mathscr{L}_{2}^{m}[0, \infty)$ and $\mathscr{Y}:=\mathscr{L}_{2}^{p}[0, \infty)$ are the input and output spaces, respectively, and $\mathscr{D}(\mathbf{P})$ is the domain of $\mathbf{P}$. Similarly, let the controller $C$ be represented by a linear mapping $C: \mathscr{D}(C) \subset \mathscr{Y} \rightarrow \mathscr{U}$.

The system in Figure 1, denoted $[\mathbf{P}, C]$, is defined as stable if the operators mapping $\left(v_{1}, v_{2}, w_{1}, w_{2}\right) \mapsto(u, y)$ are bounded, whilst the plant $\mathbf{P}$ is stabilisable if there exists a controller $C$ : $\mathscr{Y} \rightarrow \mathscr{U}$ such that $[\mathbf{P}, C]$ is stable. Some basic results on the $v$-gap metric and $\mathscr{H}_{\infty}$ loop-shaping are now reviewed, followed by the extension to the spatially distributed case.

\section{$3.1 \mathscr{H}_{\infty}$ loop-shaping and the $v$-gap metric $\delta_{v}$}

The design of feedback controllers for a spatially distributed system $\mathbf{P}$ are sought, based upon a finite dimensional approximation $P$ obtained via some appropriate means of spatial discretization. As the fidelity of the spatial discretization increases, the poles of $P$ tend to converge to locations in the complex plane, but in doing so may cross the imaginary axis. It is therefore important that the closed-loop system tolerate uncertainty in the number of right-half plane poles of $P$, as well as the poorly resolved and unmodelled high frequency dynamics inherent in any spatial discretization. These types of uncertainty are captured by augmenting the normalized coprime factors of $P$ with perturbations $\Delta_{N}, \Delta_{M} \in \mathscr{R} \mathscr{H}_{\infty}$ to form the perturbed model:

$$
P_{\text {pert }}=\left(N+\Delta_{N}\right)\left(M+\Delta_{M}\right)^{-1}:\left\|\left[\begin{array}{c}
\Delta_{N} \\
\Delta_{M}
\end{array}\right]\right\|_{\infty}<\frac{1}{\gamma}
$$

where $P=N M^{-1}$ and $\gamma>1$. Referring to Figure 1 (and replacing $\mathbf{P}$ with $P$ ), it can be shown (Vinnicombe 2001, pp. 61-62) that it is the infinity-norm of the transfer function from disturbances $v_{1}$ and $v_{2}$ (or $w_{1}$ and $w_{2}$ ), to measurements $y$ and control inputs $u$, that should be minimised in order to obtain robust stability with respect to perturbations to the normalized coprime factors of the model. Hence, the stability margin for coprime factor perturbations $b_{P, C}$ is de- fined as follows (Vinnicombe 2001):

$$
b_{P, C}:= \begin{cases}\left\|\left[\begin{array}{l}
P \\
I
\end{array}\right](I-C P)^{-1}[-C I]\right\|_{\infty}^{-1} & \text { if }[P, C] \text { is stable } \\
0 & \text { otherwise. }\end{cases}
$$

It follows that $b_{P, C} \geq \frac{1}{\gamma}$ and so a natural objective is to make $b_{P, C}$ as large as possible, subject to design criteria (McFarlane \& Glover 1992). By way of illustration, for SISO systems it can be shown (Vinnicombe 2001, Thm 2.10) that a feedback system with $b_{P, C}=0.3$ provides reasonable gain and phase margins (Åström \& Murray 2008, p. 281) of at least 2 and $35^{\circ}$, respectively.

Coprime factor perturbations are not unique, that is, given $N, M$ and $P_{\text {pert }}$, there are many $\Delta_{N}, \Delta_{M}$ for which $P_{\text {pert }}=\left(N+\Delta_{N}\right)\left(M+\Delta_{M}\right)^{-1}$ and so $b_{P, C}$ can be viewed as a radius of the largest 'ball' of plants stabilized by $C$. An appropriate metric in this case is the $v$-gap metric. Defining $\Psi(\cdot, \cdot): \mathscr{R}^{p \times m} \times \mathscr{R}^{p \times m} \rightarrow \mathscr{R}^{p \times m}$ as:

$$
\Psi\left(P_{1}, P_{2}\right):=\left(I+P_{2} P_{2}^{*}\right)^{-1 / 2}\left(P_{1}-P_{2}\right)\left(I+P_{1}^{*} P_{1}\right)^{-1 / 2},
$$

then the $v$-gap metric between two plants $\delta_{v}(\cdot, \cdot): \mathscr{R}^{p \times m} \times$ $\mathscr{R}^{p \times m} \rightarrow \mathbb{R}$ can be defined as follows (Zhou \& Doyle 1998, Thm 17.6):

$$
\delta_{v}\left(P_{1}, P_{2}\right):= \begin{cases}\left\|\Psi\left(P_{1}, P_{2}\right)\right\|_{\infty}, & \text { if } \operatorname{det}\left(I+P_{2}^{*} P_{1}\right)(j \omega) \neq 0 \quad \forall \omega \in \mathbb{R} \\ & \text { and } \operatorname{wno} \operatorname{det}\left(I+P_{2}^{*} P_{1}\right)+\eta\left(P_{1}\right) \\ 1, & \text { otherwise, }\end{cases}
$$

where $\eta(P)$ and $\eta_{0}(P)$ denote the number of open right-half plane and imaginary axis poles, respectively, of $P(s)$. The winding number wno $(g)$ is the number of anti-clockwise encirclements around the origin of a scalar transfer function $g(s)$, as $s$ follows the standard Nyquist D-contour.

Thus, one of the main advantages of the $v$-gap over the $\mathscr{H}_{2}$ gap metric is that it can be interpreted in terms of the transfer functions of two systems, as opposed to their coprime factorisations, a fact that will be exploited in the next section. The other advantage of the $v$-gap is that it is typically less conservative than the $\mathscr{H}_{2}$-gap, i.e. $\delta_{v}\left(P_{1}, P_{2}\right) \leq$ $\delta_{g}\left(P_{1}, P_{2}\right)$ (Vinnicombe 2001, Thm 7.5).

The $v$-gap measures the difference between two plants from a closed-loop, as opposed to an open-loop perspective. In particular, the following is true (Vinnicombe 2001, Thm 3.8):

$$
b_{P_{2}, C} \geq b_{P_{1}, C}-\delta_{v}\left(P_{1}, P_{2}\right) .
$$

Hence, if $C$ performs well with plant $P_{1}$, and the difference between $P_{1}$ and $P_{2}$ is sufficiently small, then $C$ is guaranteed to achieve a certain level of performance with $P_{2}$. 
Like the $\mathscr{H}_{2}$ gap-metric, the $v$-gap is also a metric on the space $\mathscr{R}^{p \times m}$ (Vinnicombe 2001, Cor. 3.4), and therefore satisfies the following triangle inequality:

$$
\delta_{v}\left(P_{1}, P_{3}\right) \leq \delta_{v}\left(P_{1}, P_{2}\right)+\delta_{v}\left(P_{2}, P_{3}\right)
$$

This is used in the next section to construct a bound on the $v$-gap between a spatially distributed plant and a finitedimensional approximation.

Next, results are reviewed that show the $v$-gap is welldefined for the class of infinite-dimensional systems that can be approximated by rationals.

\subsection{The infinite dimensional case}

As in Georgiou \& Smith (1992), define $\mathscr{C}_{0}$ as the class of functions continuous on $j \mathbb{R} \cup\{\infty\}$ and $\mathscr{A}_{0}:=\mathscr{H}_{\infty} \cap \mathscr{C}_{0}$. Note that $\mathscr{A}_{0}$ is the completion of $\mathscr{R} \mathscr{H}_{\infty}$ and so there exists a sequence $\left\{A_{i}\right\} \in \mathscr{R} \mathscr{H}_{\infty}$ such that $A_{i} \rightarrow \mathbf{A}$ if and only if $\mathbf{A} \in \mathscr{A}_{0}$ (Vinnicombe 2001, p. 236). Let $\mathscr{P} p \times m$ denote the class of all $m$-input, $p$-output LTI systems that are stabilisable, then the class $\mathscr{A}^{p \times m}$ is defined to be the set of all plants $\mathbf{P} \in \mathscr{P}^{p \times m}$ with transfer functions $\boldsymbol{P}:=$ $\boldsymbol{N} \boldsymbol{M}^{-1}=\tilde{\boldsymbol{M}}^{-1} \tilde{\boldsymbol{N}}$, and $\boldsymbol{G}=\left[\begin{array}{l}\boldsymbol{M} \\ \boldsymbol{N}\end{array}\right]$ such that $\boldsymbol{G}^{*} \boldsymbol{G}=\boldsymbol{I}$, with entries in $\mathscr{A}_{0}$ (Georgiou \& Smith 1992). The fact that $\mathbf{P} \in$ $\mathscr{A}^{p \times m}$ possesses a continuous coprime factorisation enables the computation of the $v$-gap between systems in $\mathscr{A}^{p \times m}$ and systems belonging to $\mathscr{R}^{p \times m}$ (Vinnicombe 2001, p. 236). Thus, the ideas presented in this paper can be extended to other systems that can be approximated by rationals, e.g. systems with time delays.

\section{Design procedure}

The proposed design procedure for selecting a low order model, suitable for control of an LTI spatially distributed system, is summarised below:

(i) Begin by spatially discretising the plant via some appropriate method e.g. finite differencing, on grids of varying resolution, to ascertain the approximate locations of any right-half plane poles and/or zeros.

(ii) In view of the above, assess the closed-loop design specifications to establish the closed-loop bandwidth $\omega_{b}$ and a desirable loop-shape $P_{\text {target }}$ for the singular values of the open-loop system.

(iii) Spatially discretize the system on a low number $n$ of grid-points, and compute the Laplace transform of the resulting low-order finite-dimensional system to obtain the transfer function $P_{n}$.

(iv) Plot the singular values of $P_{n}$ against frequencies around $\omega_{b}$.

(v) Repeat steps (iii) and (iv), each time increasing $n$ until the singular value plots of $P_{n}$ around $\omega_{b}$ don't change much, at which point fix $n$ and thus $P_{n}$. (vi) Design pre- and/or post-compensators, $W_{i}$ and $W_{o}$, respectively, to obtain (or at least approximate) the desired loop shape $P_{\text {target }}=W_{o} P_{n} W_{i}$.

(vii) Starting from some low value for $n$, compute the $v$-gaps between weighted plants of successively higher spatial discretization to produce a plot of $\delta_{v}\left(W_{o} P_{n} W_{i}, W_{o} P_{n+1} W_{i}\right)$ against $n$. Stop when $\delta_{v}\left(W_{o} P_{n} W_{i}, W_{o} P_{n+1} W_{i}\right)<\mu$, where $\mu \in \mathbb{R}$ is sufficiently small for one to assume the sequence $\left\{\delta_{v}\left(W_{o} P_{n} W_{i}, W_{o} P_{n+1} W_{i}\right)\right\}$ is asymptoting towards zero. The validity of this assumption is discussed in Remark 6.

(viii) Construct and plot a sequence $\left\{a_{n}\right\}$ that upper bounds the plot from (vii) for $n \geq n_{0}$ and satisfies $\sum_{n=n_{0}}^{\infty} a_{n}<\infty$. $n_{0}$ should be chosen such that $\sum_{n=n_{0}}^{\infty} a_{n}=\vartheta \leq \vartheta_{\max }$ where $\vartheta_{\max }$ is a userspecified upper bound on $\delta_{v}\left(W_{o} P_{n_{0}} W_{i}, W_{o} \boldsymbol{P} W_{i}\right)$, the $v$ gap between the weighted model and weighted plant. In addition, the sequence $\left\{a_{n}\right\}$ should be chosen so that its sum to infinity is easy to calculate.

(ix) Synthesize a loop-shaping controller $C$ to obtain the optimal stability margin $b_{\text {opt }}\left(W_{o} P_{n_{0}} W_{i}\right)$.

(x) Specify $0<b_{\text {opt }}\left(W_{o} \boldsymbol{P} W_{i}\right)<1$. With respect to (4), if $b_{\text {opt }}\left(W_{o} P_{n_{0}} W_{i}\right)-\vartheta \leq b_{\text {opt }}\left(W_{o} \boldsymbol{P} W_{i}\right)$ then either return to step (ii) and change $P_{\text {target }}$ in such a way as to make $b_{\text {opt }}\left(W_{o} P_{n_{0}} W_{i}\right)$ greater, and/or choose $n_{0}$ that satisfies a smaller value of $\vartheta_{\max }$ in step (viii) above.

Remark 1 As the resolution of a spatial discretization is increased, extra eigenvalues are generated, which generally converge to specific locations within the complex plane (Trefethen \& Embree 2005, p. 407). If the underlying system possesses some damping/diffusion, then these additional eigenvalues will typically appear further into the left half-plane (Trefethen 2000, p. 152). Physically, this corresponds to resolving the faster dynamics associated with smaller spatial scales. Broadly speaking, the desired closedloop bandwidth defines the temporal frequency range that should be modelled, which in turn corresponds to a particular level of spatial discretization. Therefore, the required spatial resolution (steps (vii)-(x)) depends on the weighted plant, since the compensators (computed in steps (i)-(vi)) determine the bandwidth of the system. In other words, it is the $v$-gap between weighted (rather than unweighted) model and plant that is of relevance here.

Remark 2 In order to set $\omega_{b}$, it is necessary first to determine the approximate locations of any right-half plane poles and/or zeros (step (i)). The systems that arise from spatial discretizations are typically large and sparse. Efficient algorithms for computing the eigenvalues (poles) of such systems exist (e.g. Lehoucq, Sorensen \& Yang (1998)) and are available in standard linear algebra packages. The system zeros, on the other hand, are typically computed as the solutions of a generalised eigenvalue problem (Skogestad \& Postlethwaite 2005, p. 138), for which sparse matrix techniques do not yet exist. In addition, it is known that the system zeros of rational approximations can be significantly different to the exact zeros (Curtain \& Morris 2009, Cheng 
\& Morris 2003). Care must therefore be exercised, particularly if the underlying system possesses zeros on, or close to the imaginary axis, since the movement of zeros across the imaginary axis induces a large change in the $v$-gap metric.

To complete this section the theoretical justification for steps (vii)-(viii) is provided by showing that, with some mild assumptions, (I) the $v$-gaps between models of successively finer spatial resolution $\delta_{v}\left(P_{n}, P_{n+1}\right)$ converges to zero as $n \rightarrow \infty$, (II) the order of convergence is bounded by the order of convergence of $\left\{\left\|P_{n}-P_{n+1}\right\|_{\infty}\right\}$, (III) $\left\{\delta_{v}\left(P_{n}, P_{n+1}\right)\right\}$ is upper-bounded by another sequence $\left\{a_{n}\right\}$ that has a finite series, and (IV) there exists an $n_{0}$ such that $\sum_{n=n_{0}}^{\infty} a_{n} \geq \delta_{v}\left(P_{n_{0}}, \boldsymbol{P}\right)$.

Proposition 3 Assume there exists an $n_{0} \in \mathbb{N}$ such that for all $n \geq n_{0}$, (i) $\delta_{v}\left(P_{n}, \boldsymbol{P}\right) \neq 1$, (ii) successive discretization of a stable spatially distributed system $\mathbf{P} \in \mathscr{A}^{p \times m}$ creates a sequence $\left\{P_{n}\right\} \in \mathscr{R}^{p \times m}$ such that $\left\|P_{n}-\boldsymbol{P}\right\|_{\infty} \stackrel{n \rightarrow \infty}{\longrightarrow} 0$. Then $\delta_{v}\left(P_{n}, P_{n+1}\right) \stackrel{n \rightarrow \infty}{\longrightarrow} 0$.

PROOF. If there exists an $n_{0} \in \mathbb{N}$ such that $\delta_{v}\left(P_{n}, \boldsymbol{P}\right) \neq 1$ for all $n \geq n_{0}$ then (3) and the submultiplicative property of the infinity norm can be used to write:

$$
\delta_{V}\left(P_{n}, \boldsymbol{P}\right) \leq\left\|\left(\boldsymbol{I}+\boldsymbol{P} \boldsymbol{P}^{*}\right)^{-\frac{1}{2}}\right\|_{\infty}\left\|\left(I+P_{n}^{*} P_{n}\right)^{-\frac{1}{2}}\right\|_{\infty}\left\|P_{n}-\boldsymbol{P}\right\|_{\infty} .
$$

Since the (self-adjoint) operators $\boldsymbol{P P}^{*}$ and $P_{n}^{*} P_{n}$ are positive semidefinite, i.e. $\boldsymbol{P} \boldsymbol{P}^{*} \geq 0$ and $P_{n}^{*} P_{n} \geq 0$, it follows that $\left\|\left(\boldsymbol{I}+\boldsymbol{P} \boldsymbol{P}^{*}\right)^{-\frac{1}{2}}\right\|_{\infty} \leq 1$ and $\left\|\left(I+P_{n}^{*} P_{n}\right)^{-\frac{1}{2}}\right\|_{\infty} \leq 1$. Therefore $\delta_{v}\left(P_{n}, \boldsymbol{P}\right) \leq\left\|P_{n}-\boldsymbol{P}\right\|_{\infty}$, from which it is clear that if $\left\|P_{n}-\boldsymbol{P}\right\|_{\infty} \stackrel{n \rightarrow \infty}{\longrightarrow} 0$, then $\delta_{v}\left(P_{n}, \boldsymbol{P}\right) \stackrel{n \rightarrow \infty}{\longrightarrow} 0$. Replacing $\boldsymbol{P}$ with $P_{n+1}$ yields $\delta_{v}\left(P_{n}, P_{n+1}\right) \leq\left\|P_{n}-P_{n+1}\right\|_{\infty}$, and since any convergent sequence in a Hilbert space is Cauchy (Dullerud \& Paganini 2000, p. 106), it follows that if $\left\|P_{n}-\boldsymbol{P}\right\|_{\infty} \stackrel{n \rightarrow \infty}{\longrightarrow} 0$, then $\left\|P_{n}-P_{n+1}\right\|_{\infty} \stackrel{n \rightarrow \infty}{\longrightarrow} 0$ and so $\delta_{V}\left(P_{n}, P_{n+1}\right) \stackrel{n \rightarrow \infty}{\longrightarrow} 0$.

The first assumption in Proposition 3 is necessary to ensure that the determinant and winding number conditions in (3) are satisfied upon refinement of the spatial discretization.

The following proposition shows that the sequence of $v$ gaps between plant models converges at least as fast as the sequence of infinity-norm differences.

Proposition 4 Assume $\left\{\left\|P_{n}-P_{n+1}\right\|_{\infty}\right\}$ converges with order $\alpha>0$, then $\left\{\delta_{v}\left(P_{n}, P_{n+1}\right)\right\}$ converges with order $\beta \geq \alpha$.

PROOF. If $\left\{\left\|P_{n}-P_{n+1}\right\|_{\infty}\right\}$ converges with order $\alpha$, then for an $n_{0} \in \mathbb{N}$ there exists $k_{\alpha} \in \mathbb{R}_{+}$such that $\left\|P_{n}-P_{n+1}\right\|_{\infty} \leq$ $k_{\alpha} n^{-\alpha} \forall n \geq n_{0}$. From the proof of Proposition 3, since $\delta_{v}\left(P_{n}, P_{n+1}\right) \leq\left\|P_{n}-P_{n+1}\right\|_{\infty}$, then $\delta_{v}\left(P_{n}, P_{n+1}\right) \leq$ $k_{\beta} n^{-\beta} \leq k_{\alpha} n^{-\alpha}$ for some $k_{\beta} \in \mathbb{R}_{+}$.
Theorem 5 Assume the sequence $\left\{\delta_{v}\left(P_{n}, P_{n+1}\right)\right\}$ converges with order $\alpha>1$, then there exists an $n_{0} \in \mathbb{N}$ and a sequence $\left\{a_{n}\right\}$ such that $a_{n} \geq \delta_{v}\left(P_{n}, P_{n+1}\right)$ for all $n \geq n_{0}$, and $\delta_{v}\left(\boldsymbol{P}, P_{n_{0}}\right) \leq \sum_{n=n_{0}}^{\infty} a_{n}<1$.

PROOF. From the proof of Proposition 4, $\delta_{v}\left(P_{n}, P_{n+1}\right) \leq$ $k_{\alpha} n^{-\alpha}$ for $n \geq n_{0}$, so $a_{n}:=k_{\alpha} n^{-\alpha}$ satisfies $a_{n} \geq \delta_{v}\left(P_{n}, P_{n+1}\right)$. Using the integral convergence test, it can be shown that $\sum_{n=n_{0}}^{\infty} a_{n}<\infty$ for $\alpha>1$. Since the $v$-gap satisfies a triangle inequality, it follows that:

$$
\delta_{v}\left(P_{n_{0}}, \boldsymbol{P}\right) \leq \sum_{n=n_{0}}^{\infty} \delta_{v}\left(P_{n}, P_{n+1}\right) \leq \sum_{n=n_{0}}^{\infty} a_{n}
$$

For the sequence $\left\{a_{n}\right\}$ to be of any practical use, it is required that $\sum_{n=n_{0}}^{\infty} a_{n}<1$. Since $\sum_{n=n_{0}}^{\infty} a_{n} \stackrel{n_{0} \rightarrow \infty}{\longrightarrow} 0, n_{0}$ may be taken sufficiently large to ensure that $\sum_{n=n_{0}}^{\infty} a_{n}<1$.

Remark 6 The preceding theorem justifies the construction of a bounding sequence whose sum to infinity upper bounds the $v$-gap between a spatially distributed plant and a loworder approximation. At present, it is necessary to assume that if $\left\{a_{n}\right\}$ bounds $\left\{\delta_{v}\left(W_{o} P_{n} W_{i}, W_{o} P_{n+1} W_{i}\right)\right\}$ over the range of $n$ used in step (vii), then it will continue to bound this $v$ gap sequence for all higher values of $n$. This is not an unreasonable assumption to make, since unresolved spatial scales generally correspond to unresolved temporal dynamics in higher frequency ranges, as discussed in Remark 1. Provided these dynamics occur at frequencies above the closedloop bandwidth $\omega_{b}$, then sensibly designed compensators (i.e. ones that provide the shaped plant with a steep roll-off above $\omega_{b}$ ) should ensure the closed-loop system is insensitive to this high frequency uncertainty, and thus prevent the $v$-gap sequence from increasing in value upon spatial refinement. As far as is practically feasible, one should compute $\left\{\delta_{v}\left(W_{o} P_{n} W_{i}, W_{o} P_{n+1} W_{i}\right)\right\}$ for the largest values of $n$ that time and computational resources allow, in order to be as certain as possible that this sequence is asymptoting towards zero.

For unstable plants, convergence of the graph symbols of transfer functions can be considered.

Proposition 7 Assume there exists an $n_{0} \in \mathbb{N}$ such that for all $n \geq n_{0}$, (i) $\delta_{v}\left(P_{n}, \boldsymbol{P}\right) \neq 1$, (ii) successive discretization of a spatially distributed system $\mathbf{P} \in \mathscr{A}^{p \times m}$ creates a sequence $\left\{G_{n}\right\} \in \mathscr{R} \mathscr{H}_{\infty}^{(p \times m) \times m}$ such that $\left\|G_{n}-\boldsymbol{G}\right\|_{\infty} \stackrel{n \rightarrow \infty}{\longrightarrow} 0$. Then $\delta_{v}\left(P_{n}, P_{n+1}\right) \stackrel{n \rightarrow \infty}{\longrightarrow} 0$.

PROOF. From (Georgiou \& Smith 1992, Prop 1) it is shown that $\delta_{g}\left(P_{n}, \boldsymbol{P}\right) \leq\left\|G_{n}-\boldsymbol{G}\right\|_{\infty}$. Since $\delta_{v}\left(P_{n}, \boldsymbol{P}\right) \leq$ $\delta_{g}\left(P_{n}, \boldsymbol{P}\right)$ (Vinnicombe 2001, Thm 7.5), then $\delta_{v}\left(P_{n}, \boldsymbol{P}\right) \leq$ $\left\|G_{n}-\boldsymbol{G}\right\|_{\infty}$. Therefore $\delta_{v}\left(P_{n}, \boldsymbol{P}\right) \stackrel{n \rightarrow \infty}{\longrightarrow} 0$. Replacing $\boldsymbol{P}$ with $P_{n+1}$ yields $\delta_{v}\left(P_{n}, P_{n+1}\right) \leq\left\|G_{n}-G_{n+1}\right\|_{\infty}$, and again, 
if $\left\|G_{n}-\boldsymbol{G}\right\|_{\infty} \stackrel{n \rightarrow \infty}{\longrightarrow} 0$, then $\left\|G_{n}-G_{n+1}\right\|_{\infty} \stackrel{n \rightarrow \infty}{\longrightarrow} 0$ and so $\delta_{v}\left(P_{n}, P_{n+1}\right) \stackrel{n \rightarrow \infty}{\longrightarrow} 0$.

Sufficient conditions for the convergence of transfer functions in the $\mathscr{H}_{\infty}$-norm for stable systems, and in the $\mathscr{H}_{2}$ gap metric for unstable systems are discussed in Morris (1994). Also, it is worth noting that the choice of spatial discretization technique influences the order of convergence of $\left\{\delta_{v}\left(P_{n}, P_{n+1}\right)\right\}$.

The following section demonstrates the design procedure.

\section{Example: The 1D heat equation}

Consider the following nondimensionalised heat equation in a medium of one spatial dimension (Boskovic, Krstic \& Liu 2001) with a measurement of temperature gradient at one end:

$$
\begin{aligned}
\frac{\partial \mathbf{q}(x, t)}{\partial t} & =\frac{\partial^{2} \mathbf{q}(x, t)}{\partial x^{2}}+\lambda \mathbf{q}(x, t), \quad x \in \Omega, \\
y(t) & =\left.\frac{\partial \mathbf{q}(x, t)}{\partial x}\right|_{x=-1},
\end{aligned}
$$

with initial and boundary conditions:

$$
\begin{aligned}
\mathbf{q}(x, 0) & =\mathbf{q}_{0}(x), \\
\frac{\partial \mathbf{q}}{\partial t}(-1, t) & =\frac{-1}{\tau_{u}} \mathbf{q}(-1, t)+\frac{1}{\tau_{u}} u(t), \\
\frac{\partial \mathbf{q}}{\partial t}(+1, t) & =\frac{-1}{\tau_{d}} \mathbf{q}(+1, t)+\frac{1}{\tau_{d}} d(t),
\end{aligned}
$$

where the temperature of the medium is $\mathbf{q}(\cdot, \cdot): \Omega \times \mathbb{R}_{+} \rightarrow \mathbb{R}$, and $\lambda \in \mathbb{R}$ is a parameter that accounts for the internal heating of the material. $\Omega \in[-1,1]$ is a bounded domain with lower and upper boundaries $\partial \Omega_{-1}=-1$ and $\partial \Omega_{+1}=1$, respectively, $x \in \Omega$ is a point within the domain and $y(\cdot)$ : $\mathbb{R}_{+} \rightarrow \mathbb{R}$ is the temperature gradient at the lower boundary. A control input $u(\cdot): \mathbb{R}_{+} \rightarrow \mathbb{R}$ is applied to a heating element with time constant $\tau_{u}$ at the lower boundary, whilst a disturbance input $d(\cdot): \mathbb{R}_{+} \rightarrow \mathbb{R}$ with time constant $\tau_{d}$ enters at the upper boundary. For the present example $\tau_{u}=\tau_{d}=1$.

The interesting property of $(6 a)$ is that under homogenous Dirichlet boundary conditions $(\mathbf{q}(-1, t)=\mathbf{q}(+1, t)=0)$, it can be shown (via separation of variables) that the system is unstable for $\lambda>\pi^{2} / 4$. For the present example $\lambda=2.39$ so that the (infinite-dimensional) system is stable.

Computationally, this infinite dimensional system is not of immediate use. The system is therefore spatially discretized on a grid of $n_{x}$ grid-points using an appropriate method. For the sake of illustration, second order finite differences are employed on a grid $\left\{x_{1}, \ldots, x_{n_{x}}\right\}$, where $x_{j}:=1-(j-$ 1) $\Delta x$ for $j=1, \ldots, n_{x}$, and the grid spacing $\Delta x:=2 /\left(n_{x}-\right.$
$1)$ is constant. The corresponding vector of temperatures is defined as $q(t):=\left[q_{1}(t) \cdots q_{n_{x}}(t)\right]^{T}$, such that $q_{j}(t):=$ $\mathbf{q}\left(x_{j}, t\right)$ for $j=1, \ldots, n_{x}$. The first order spatial derivative operator is approximated by the following differentiation matrix:

$$
\frac{\partial}{\partial x} \approx X \in \mathbb{R}^{n_{x} \times n_{x}}:=\frac{1}{2 \Delta x}\left[\begin{array}{ccccc}
3 & -4 & 1 & & \\
-1 & 0 & 1 & & \\
& \ddots & \ddots & \ddots & \\
& -1 & 0 & 1 \\
& -1 & 4 & -3
\end{array}\right]
$$

Likewise for the second order spatial derivative operator:

$$
\frac{\partial^{2}}{\partial x^{2}} \approx Y \in \mathbb{R}^{n_{x} \times n_{x}}:=\frac{1}{\Delta x^{2}}\left[\begin{array}{ccccc}
-2 & 1 & & & \\
1 & -2 & 1 & & \\
& \ddots & \ddots & \ddots & \\
& & 1 & -2 & 1 \\
& & & 1 & -2
\end{array}\right]
$$

Inserting (7) into (6a) and (6b) with the boundary conditions (6d)-(6e) yields the following LTI state-space system:

$$
\begin{aligned}
& \frac{d}{d t} q(t)=\underbrace{\left[\begin{array}{ccc}
-1 & 0_{1 \times\left(n_{x}-2\right)} & 0 \\
Y_{2: n_{x}-1,1} & Y_{2: n_{x}-1,2: n_{x}-1}+\lambda I_{\left(n_{x}-2\right) \times\left(n_{x}-2\right)} & Y_{2: n_{x}-1, n_{x}} \\
0 & 0_{1 \times\left(n_{x}-2\right)} & -1
\end{array}\right]}_{A} q(t) \\
& +\underbrace{\left[\begin{array}{c}
0 \\
0_{\left(n_{x}-2\right) \times 1} \\
1
\end{array}\right]}_{B_{u}} u(t)+\underbrace{\left[\begin{array}{c}
1 \\
0_{\left(n_{x}-2\right) \times 1} \\
0
\end{array}\right]}_{B_{d}} d(t) \\
& y(t)=\underbrace{X_{n_{x}, 1: n_{x}}}_{C} q(t) .
\end{aligned}
$$

where the notation $Z_{a: b, c: d}$ is to be interpreted as 'rows $a$ to $b$ and columns $c$ to $d$ of matrix $Z$ '. Notice how the boundary conditions (6d)-(6e) are enforced by modifying the top and bottom rows of $Y$, and how the matrices $B_{u}$ and $B_{d}$ reflect the fact that the inputs enter at the boundaries only. This (numerical) method of enforcing boundary conditions is commonly known as boundary-bordering (Boyd 2001, p. 111). The crucial question is what value of $n_{x}$ should be used in order to obtain a 'good' model for controller design? The answer lies in the control objective, which in the present case is to attenuate a constant disturbance $d(t)=1$ on the output $y(t)$.

Taking the Laplace Transform of (8) between control input $u(t)$ and measured output $y(t)$ yields:

$$
y(s)=\underbrace{C(s I-A)^{-1} B_{u}}_{P_{n_{x}}} u(s) .
$$

In order to reject output disturbances, high gain in some low frequency range is required. Since $P_{100}$ has a righthalf plane zero at $\omega \approx 1.8 \mathrm{rad} / \mathrm{s}$ then the bandwidth of the closed-loop system $\omega_{b}$ must be set lower, in the present case at $\omega_{b}=0.5 \mathrm{rad} / \mathrm{s}$. By designing the closed-loop to attenuate all frequencies above $\omega_{b}$, a reduction in the sensitivity of the 

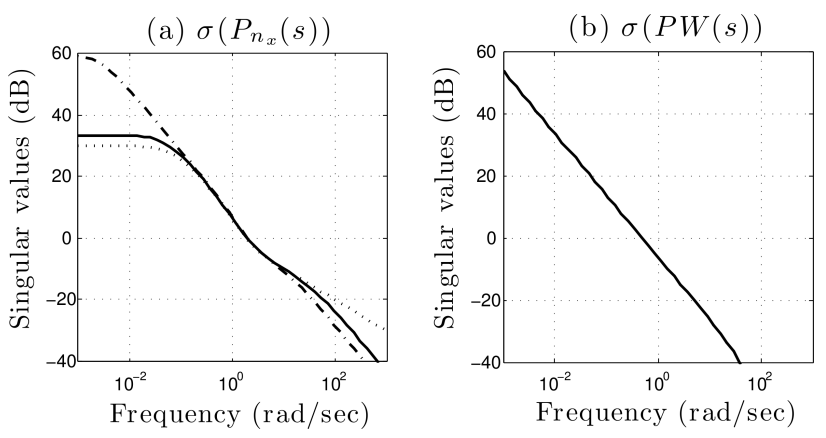

Fig. 2. Open-loop singular-value plots of (a) $P_{n_{x}}$ for $n_{x}=6(\cdot-), n_{x}=10(-), n_{x}=100(\cdot)$, and (b) $P W$.

closed loop to plant/model error in that frequency range is achieved. In other words, the model is only required to be 'accurate' up to around $\omega_{b}$. Figure 2(a) shows the singular value plots of $P_{n_{x}}$ for three different resolutions $n_{x}$.

Figure 2(a) reveals that a model of resolution $n_{x}=10$ is reasonably accurate up to and around $\omega_{b}$. Denoting this model $P_{10}$, a precompensator $W$ is then designed to achieve a typical desired loop shape $P W$; in this case one with a crossover frequency at $\omega_{b}=0.5$, a crossover slope of approximately $-20 \mathrm{~dB} /$ decade, rejection of constant disturbances and $-40 \mathrm{~dB} /$ decade role-off at high frequencies. Such a loop is shown in Figure 2(b) and is defined as follows:

$$
P_{\text {target }}(s)=P_{n_{x}} W(s):=\frac{1}{2 s(s / 50+1)} .
$$

Given this loop-shape and plant model $P_{10}, W$ can be computed using MATLAB's loopsyn comand. At this point a loop-shaping controller for the shaped plant $P_{10} W$ could be computed, but there would be no guarantee of it working on $\boldsymbol{P} W$, and nor would it be known how lower fidelity models might perform. To address these issues step (vii) is implemented by plotting the $\boldsymbol{v}$-gaps of weighted models of successively higher spatial fidelity, as shown in Figure 3. In addition to finite-differencing, the $\boldsymbol{v}$-gaps between models obtained using Chebyshev differentiation matrices (Weideman $\&$ Reddy 2000) are also computed, and clearly show how, from a closed-loop perspective, different methods of spatial discretization can yield models that converge faster to the weighted plant. With respect to the finite differencing curve, a sequence is constructed that upper bounds the $v$-gap curve above a certain value of $n_{x}$. Referring to Figure 3 , for $6 \leq n_{x} \leq 25$ a sequence such as $\left\{a_{n_{x}}\right\}:=2.05 /\left(n_{x}\left(n_{x}+1\right)\right)$ upper bounds the finite difference curve, and since it appears to be converging to zero slower, then one can assume it is an upper bound for all $n_{x} \geq 6$. Theorem 5 can then be applied to obtain the following bound:

$$
\delta_{v}\left(P_{6} W, \boldsymbol{P W}\right) \leq \sum_{n_{x}=6}^{\infty} \frac{2.05}{n_{x}\left(n_{x}+1\right)}=\frac{2.05}{6}=0.34
$$

The actual value of $\delta_{v}\left(P_{6} W, \boldsymbol{P} W\right)$, as computed by studying the convergence of $\left\{\delta_{v}\left(P_{6} W, P_{n_{x}} W\right)\right\}$ for $n_{x} \gg 6$, is approx-

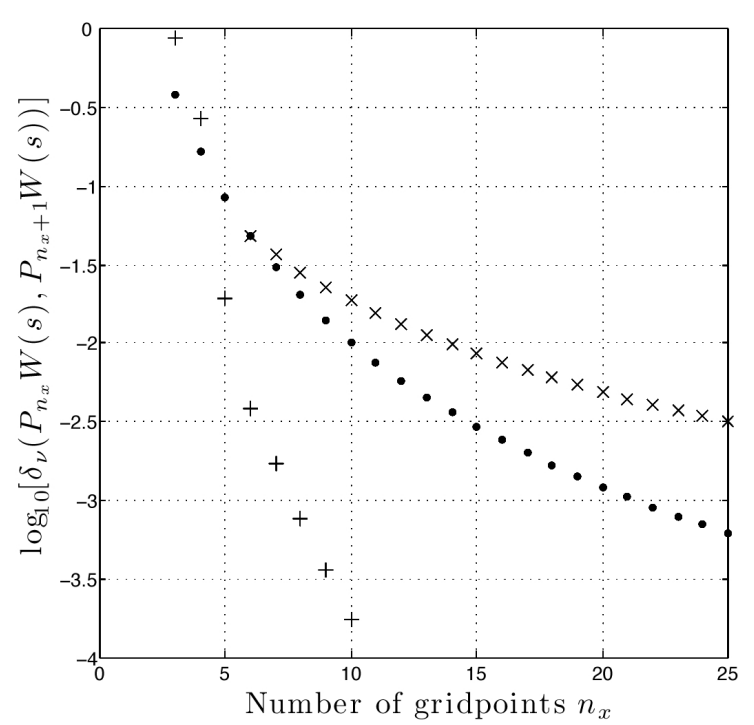

Fig. 3. $\log _{10}\left(\delta_{v}\left(P_{n_{x}} W, P_{n_{x}+1} W\right)\right)$ versus $n_{x}$ for finite-difference (.) and Chebyshev $(+)$ discretizations. Also shown is the sequence $2.05 /\left(n_{x}\left(n_{x}+1\right)\right)(\times)$ for $n_{x} \geq 6$.

imately 0.17 . However, note that this method of computing $\delta_{v}\left(P_{6} W, \boldsymbol{P} W\right)$ is expensive.

A controller $C$ is then synthesized, which achieves the optimum stability margin $b_{\text {opt }}\left(P_{6} W\right)=0.55$. From (4), $b_{P W, C} \geq$ $b_{P_{6} W, C}-\delta_{v}\left(P_{6} W, \boldsymbol{P} W\right)=0.55-0.34=0.21$. Thus, the controller synthesized on the low order model will work reasonably well on the plant. In the absence of a physical experiment, $C$ is instead implemented on a high fidelity $\left(n_{x}=100\right)$ simulation model, shown in Figure 4. At this resolution the system $P_{100}$ is stable (all poles in the left half plane).

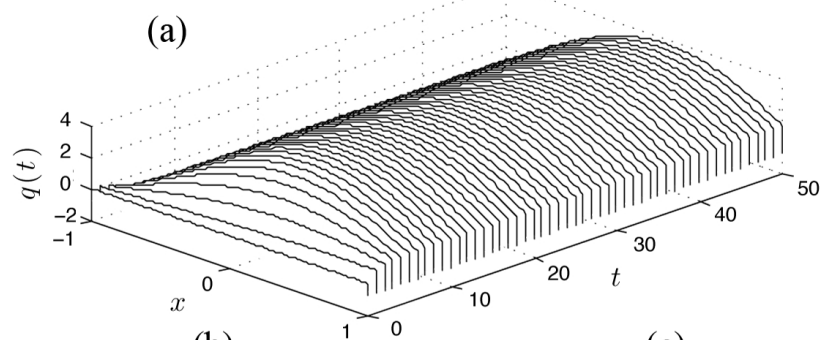

(b)
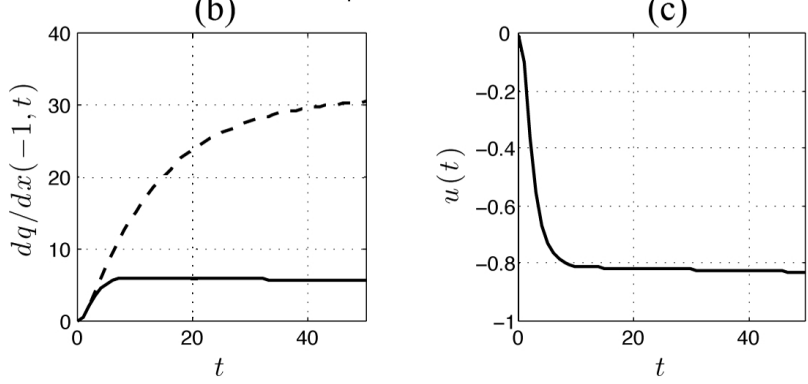

Fig. 4. (a) Simulation of the system with control for $n_{x}=100$, (b) the temperature gradients at $x=-1$ for the controlled (-) and uncontrolled (- -) cases, and (c) the control input $u(t)$. 


\section{Discussion of numerical example}

Clearly, the feedback controller, based on the low-order model $P_{6}$, has significantly reduced the effect of the disturbance on the temperature gradient at $x=-1$. If one were determined to use the lowest order model possible, one could use a different means of spatial discretization (such as Chebyshev, Fourier, etc.) and construct a less conservative bounding sequence than the one above. One could also perform a model reduction on the low-order model, and then check whether the $v$-gap between the reduced and low-order models was sufficiently small. However, in some cases it may not be desirable to reduce the system, since the states in the reduced system would not share the same physical significance as the states in the low-order model, which may be important if one wished to exploit the separation structure of the $\mathscr{H}_{\infty}$ loop-shaping controller to study the state estimates.

Interestingly in this example, the model $P_{6}$ from which the controller was synthesized is open-loop unstable, owing to a pole in the right-half plane moving across the imaginary axis as spatial resolution is increased. This clearly shows how two models (in this case $P_{100}$ and $P_{6}$ ) can differ greatly with respect to their open-loop behaviour, but are close (in terms of the $v$-gap metric) from a closed-loop perspective.

\section{Conclusions}

In this paper a new method was introduced that enabled the design of robust controllers for spatially distributed plants, based on finite-dimensional control models. It was shown how suitable control models could be obtained by computing the $v$-gaps between low-order weighted models of successively finer spatial fidelity and bounding this sequence from above by another sequence with a finite series. The triangle inequality property of the $v$-gap metric was then used to prove that such a series provided an upper bound on the $v$-gap between a weighted model in the initial sequence and the spatially distributed weighted plant.

This method is an improvement over large-scale model reduction based approaches for two reasons. Firstly, a bound on the $v$-gap between model and plant enables controllers to be synthesized that are a priori guaranteed to robustly stabilize the plant. In contrast, most model reduction methods ignore the gap between a high-order model and the plant, and may not be able to efficiently compute the gap between the high-order and reduced models. Secondly, controller synthesis based on low-order models avoids the numerical problems inherent in model reduction of large-scale systems.

Emphasis was placed on computing $v$-gaps between weighted models and plants, since the weights reflect performance criteria that directly influence the level of spatial discretization required to obtain a suitable control model. It was also shown that the order of convergence between models in terms of the $v$-gap metric is bounded by the order of convergence between the models' $\mathscr{H}_{\infty}$-norm differences. An example was presented, based on the 1D heat equation, that demonstrated the efficacy of the proposed design method.

Finally, for a bound on the $v$-gap between low-order model and plant to hold true, it was necessary to assume that if a sequence $\left\{a_{n}\right\}$ bounds $\left\{\delta_{v}\left(W_{o} P_{n} W_{i}, W_{o} P_{n+1} W_{i}\right)\right\}$ over some initial range of $n$, then it will continue to act as a bound for all higher spatial resolutions. Although it was argued that this assumption is reasonable, further research is necessary since it is not yet known for which class of system and discretization method this assumption is valid.

\section{References}

Aamo, O. M. \& Krstic, M. (2003), Flow control by feedback: Stabilization and Mixing, Springer.

Antoulas, A. C. (2005), 'An overview of approximation methods for large scale dynamical systems', Annual Reviews in Control 29, 181-190.

Åström, K. J. \& Murray, R. M. (2008), Feedback Systems: An Introduction for Scientists and Engineers, Princeton University Press.

Balas, M. J. (1978), 'Feedback control of flexible systems', IEEE Transactions on Automatic Control 23, 673-679.

Boskovic, D. M., Krstic, M. \& Liu, W. (2001), 'Boundary control of an unstable heat equation via measurement of domain-averaged temperature', IEEE Transactions on Automatic Control 46(12), 20222028.

Boyd, J. P. (2001), Chebyshev and Fourier Spectral Methods, 2 edn, Dover, Mineola, New York

Cheng, A. \& Morris, K. A. (2003), Accurate zeros approximation for infinite-dimensional systems, in 'Proceedings of the 42nd IEEE Conference on Decision and Control', Maui, USA, pp. 1315-1320.

Curtain, R. \& Morris, K. A. (2009), 'Transfer functions of distributed parameter systems: A tutorial', Automatica 45, 1101-1116.

Curtain, R. \& Zwart, H. (1995), An Introduction to Infinite-dimensional Linear Systems Theory, Springer.

Dullerud, G. \& Paganini, F. (2000), A Course in Robust Control Theory: A Convex Approach, Springer.

Georgiou, T. T. \& Smith, M. C. (1992), 'Robust stabilization in the gap metric: Controller design for distributed plants.', IEEE Transactions on Automatic Control 37, 1133-1143.

Jaimoukha, I. M. \& Kasenally, E. M. (1994), 'Krylov Subspace Methods for Solving Large Lyapunov Equations', SIAM Journal on Numerical Analysis 31(1), 227-251.

Jonckheere, E. A., Safanov, M. G. \& Silverman, L. M. (1981), Topology induced by the Hankel norm in the space of transfer matrices, in 'Proc. 20th IEEE Conference on Decision and Control including the Symposium on Adaptive Processes', Vol. 20, pp. 118-119.

Lehoucq, R. B., Sorensen, D. C. \& Yang, C. (1998), ARPACK User's Guide: Solution of Large-Scale Eigenvalue Problems with Implicitly Restarted Arnoldi Methods, SIAM Publications.

McFarlane, D. \& Glover, K. (1992), 'A loop shaping design procedure using $\mathscr{H}_{\infty}$ synthesis', IEEE Transactions on Automatic Control 37(6), 759-769.

Morris, K. A. (1994), 'Design of finite-dimensional controllers for infinitedimensional systems by approximation', Journal of Mathematical Systems, Estimation, and Control 4(2), 1-30.

Reinschke, J. (1999), $\mathscr{H}_{\infty}$-Control of spatially distributed systems, PhD thesis, Department of Engineering, University of Cambridge. 
Reinschke, J. \& Smith, M. C. (2003), 'Designing robustly stabilising controllers for LTI spatially distributed systems using coprime factor synthesis', Automatica 39, 193-203.

Rowley, C. W. (2005), 'Model reduction for fluids, using balanced proper orthogonal decomposition', International Journal of Bifurcation and Chaos 15(3), 997-1013.

Skogestad, S. \& Postlethwaite, I. (2005), Multivariable Feedback Control, John Wiley \& Sons, Ltd.

Trefethen, L. N. (2000), Spectral Methods in Matlab, SIAM.

Trefethen, L. N. \& Embree, M. (2005), Spectra and Pseudospectra The Behaviour of Nonnormal Matrices and Operators, Princeton
University Press.

Vinnicombe, G. (2001), Uncertainty and Feedback, Imperial College Press.

Weideman, J. A. C. \& Reddy, S. C. (2000), 'A MATLAB differentiation matrix suite', ACM Transactions on Mathematical Software 26(4), 465-519.

Willcox, K. \& Peraire, J. (2002), 'Balanced model reduction via the proper orthogonal decomposition', AIAA Journal 40(11).

Zhou, K. \& Doyle, J. C. (1998), Essentials of Robust Control, Prentice Hall.

Zhou, K., Doyle, J. C. \& Glover, K. (1996), Robust and optimal control, Prentice Hall. 